\title{
reviscafuences
}

ISSN: 1575-7072 | e-ISSN: 2172-7775

\section{Adaptación y desarrollo profesional docente de los especialistas de la enseñanza bilingüe}

\author{
The adaptability and professional development of teachers in bilingual education
}

\author{
(iD) Francisco Javier Sanz Trigueros \\ Universidad de Valladolid (España) \\ iD Carmen Guillén Díaz \\ Universidad de Valladolid (España)
}

\section{Resumen}

Este artículo se inscribe en la tendencia común europea de atención al desarrollo profesional y a las cualificaciones para la empleabilidad de todo individuo. Se adopta la representación de la realidad actual proporcionada por la categoría sociológica de la "modernidad líquida" (Bauman, 2007), y se consideran los efectos cualitativos de ese componente competencial procedente del mundo empresarial que es la adaptación (Fernández Aráoz, 2008), con el objetivo de dar cuenta de su valor potencial en el desarrollo profesional continuo del sector emergente de docentes responsables de la enseñanza bilingüe. Conforme a la investigación cualitativa-interpretativa se moviliza el análisis de contenido, para un tratamiento en profundidad de datos obtenidos de una muestra heterogénea de esos docentes en activo, mediante el cuestionario y el relato de vida. Los resultados revelan el estado de los componentes de esa adaptación, articulados en los rasgos de la capacidad de adaptación y los atributos de la adaptabilidad. Desde los valores presentados, se estima un bajo potencial estructural de la capacidad de adaptación y un escaso equilibrio de los atributos de la adaptabilidad. Se ofrecen pautas y estrategias de remediación orientadas a los agentes y órganos institucionales y a los formadores universitarios, a quienes corresponde atender a la mejora del desarrollo profesional de estos docentes.

\begin{abstract}
This article is part of a common European trend that focuses on the professional development and qualifications of individuals for their employability. The sociological category of "liquid modernity" (Bauman, 2007) as applied to current reality is adopted. Also, the qualitative effects of adaptability (Fernández Aráoz, 2008) as a competence derived from the business world are considered with the following aim: to account for the potential value of adaptation for the ongoing professional development of teaching specialists in bilingual education. Using qualitative-interpretative research, content analysis is performed for an in-depth treatment of data obtained from a heterogeneous sample of in-service teachers. This is done by means of questionnaires and life stories. Results reveal the status of the components of adaptation, articulated by informants as adaptive capacity features and adaptability attributes. The values presented show that adaptive capacity has low structural potential, while there is a lack of balance in adaptability attributes. Finally, guidelines and remediation strategies are offered to agents, institutional bodies and teacher trainers, responsible for the professional development of bilingual teachers.
\end{abstract}

\section{Palabras clave / Keywords}

Adaptación, Análisis de contenido, Desarrollo de la carrera, Educación bilingüe, Formación de docentes. Adaptability, Bilingual education, Career development, Content analysis, Teacher education. 


\section{Introducción}

En el seno de las recomendaciones europeas relativas a la mejora de la calidad de los sistemas de educación y formación, se asiste a los retos planteados para el horizonte 2030 -e incluso 2050 (European Commission, 2015a)-, entre los que sigue encontrándose el dominio de lenguas, ante la evidencia de que la relación entre lenguas y empleabilidad continúa incrementándose. Consecuentemente, se asume su enseñanza desde el currículo en su conjunto, en términos de desarrollo de una competencia comunicativa plurilingüe e intercultural (González Piñeiro, Guillén Díaz y Vez Jeremías, 2010), introducida en los sistemas educativos nacionales bajo la concepción de una educación plurilingüe e intercultural (Beacco et al., 2016). Una concepción que se ha llevado a cabo implantando secciones lingüísticas, en donde los programas de Aprendizaje Integrado de Contenidos y Lenguas Extranjeras (AICLE, CLIL, EMILE) constituyen el tipo de enseñanza bilingüe más utilizado.

Estos programas se nutren de numerosas aportaciones, de entre las que destacamos por su gran difusión y consenso las de Coyle, Hood y Marsh (2010). El carácter innovador de estos programas (Kiely, 2011), conlleva la problemática de la cualificación específica de especialistas de la enseñanza de lenguas extranjeras, y de la enseñanza de contenidos curriculares a través de lenguas extranjeras (Escobar Urmeneta, 2019).

Es por lo que desde diversas instancias se insiste en fortalecer este perfil profesional docente -el del profesorado de programas bilingües-e institucionalizarlo, como se viene haciendo para la profesión docente genéricamente considerada (Comisión Europea, EACEA y Eurydice, 2018; European Commission, 2015b; López Rupérez, 2014).

\section{Marco teórico}

\subsection{Estado de la cuestión}

Como en cualquier otra profesión, para este sector profesional emergente, híbrido y bivalente, que debe asumir funciones y tareas específicas, se recurre a nuevas competencias en su perfil profesional docente, dentro de los cánones de calidad (European Commission, 2019). Han incidido al respecto, entre otros, los trabajos de Pérez-Cañado (2018) y Vaillant (2014) desde los contextos universitarios. Además, como ya se aludía en Pérez Gómez, Barquín Ruiz y Angulo Rasco (1999), se asiste a un discurso que traspone a este mundo de la educación los "modelos empresariales" (p. 10).

En este sentido cabe observar, en la óptica de la categoría sociológica de "modernidad líquida" de Bauman (2007), las nuevas situaciones contractuales de estos docentes, marcadas por exigencias de cambio y renovación. Se trata de profesorado que, para mantener su funcionalidad en el sistema educativo actual, debe configurar un perfil profesional flexible, con autonomía relativa bajo la idea de construirse a sí mismo. Es lo que, en parangón con el mundo de la empresa, conduce a considerar una competencia muy demandada: la adaptación (Fernández Aráoz, 2008).

Es esta una competencia que, en la perspectiva filosófica y ontológica de Super (1980) sobre el desarrollo de la carrera, da cuenta del potencial de un individuo, de su capacidad de evolucionar. En el caso que nos ocupa, esto se traduce en la capacidad de desempeñar esas funciones y tareas nuevas generadas para la enseñanza bilingüe, ante las exigencias socio-económicas y transformaciones académico-curriculares.

Desde las teorías de las organizaciones se estima que son los "valores adaptativos" de las personas en las instituciones los que tienen un valor intrínseco (Daft, 2007), y que la adaptación requiere prácticas de introspección disponiendo de sistemas explicativos. Conforme a la teoría de los sistemas de Bertalanffy (1986), se implicaría a los sistemas comportamental y de la personalidad de los individuos por los que, respectivamente, se reconvierten, modifican, incorporan, etc., comportamientos, y se coordinan respuestas al cambio; todo ello sustentado por el compromiso con la institución.

Precisamente, el 6ํinforme EPyCE (2019) contiene el estudio sobre las competencias más demandadas en el mundo empresarial. De un marco de 18 competencias (pp. 25-26) incluidas en un cuestionario a directores de empresas multinacionales, se muestran frecuencias relativas reveladoras. La competencia denominada "Flexibilidad/Adaptación" es la más demandada, más difícil de cubrir y escasa en perfiles júnior (profesionales con 2 años de experiencia), seguida de "Compromiso". En perfiles sénior (más de 6 años de experiencia), la más demandada es "Visión estratégica", seguida de "Flexibilidad/Adaptación" que, igualmente, es la más escasa y difícil de cubrir.

Como se insiste en un reciente estudio de la Comisión Europea, EACEA y Eurydice (2018), las competencias docentes necesarias para lograr mejores resultados en los aprendizajes han de atender al "desarrollo 
profesional de los profesores" (p. 78). Se trata -considerando los marcos de competencias reunidos por Bleichenbacher (2019) - de promover la capacidad de actuar, el empoderamiento y la responsabilidad del profesorado. Según se desprende de las políticas sectoriales de la Unión Europea -la lingüística, la educativa, de empleo y de calidad-, esta formación ha de atender a un desarrollo profesional docente continuo, con el carácter de construcción progresiva de la carrera articulada en etapas. Etapas cuyas denominaciones, acuñadas por Sanz Trigueros (2018) ante las propuestas expertas, corresponden a períodos de docencia: Ingreso a la profesión (0-6 años); Transición (6-12 años); Estabilización y compromiso (12-18 años); Crisis de la carrera (18-24 años); Preparación a la jubilación (más de 24 años).

\subsection{La adaptación: sus rasgos y atributos para el desarrollo profesional docente}

La adaptación, traspuesta al ámbito educativo, se aborda como concepto nómada al servicio del área de conocimiento de Didáctica de la Lengua y la Literatura (Puren, 1998), dado que esta participa de pleno derecho en la investigación sobre la formación inicial y continua del profesorado de lenguas en las titulaciones otorgadas en la Educación Superior.

En función de los conceptos de cualificación profesional y empleabilidad, y en la perspectiva de la complejidad de Morin (2015), la adaptación se aborda como condición primera y general de toda existencia (profesional docente). En este sentido, del resumen de definiciones interdisciplinares que ha aportado Simonet (2009), es pertinente considerar en cada docente, que la adaptación:

- Se contempla como "capacidad de adaptación" a través de "rasgos" genéricos en los que reside la adaptación en potencia; y como "adaptabilidad" (Hirschi, 2015; Parsons et al., 2011; Savickas, 2005) a través de "atributos" específicos personales en los que reside la adaptación en acto.

- Resulta de la integración de esos rasgos y atributos que implican a los sistemas comportamental y de la personalidad, referidos a una identidad/yo profesional de hechos y sentimientos.

- Actúa como catalizador en la construcción del perfil profesional, pues esos rasgos y atributos, desde su complementariedad psico-socio-cognitiva y profesionalizadora, podrían resultar compensatorios.

- Es evolutiva, procesual en dependencia de etapas temporales e historias de vida, y en gran medida endógena, según las acciones realizadas para responder a los cambios.

- Se activa por tomas de decisión basadas en el compromiso con el conocimiento, los destinatarios de las enseñanzas, la profesión docente y el propio desarrollo profesional.

La definición de los rasgos de la capacidad de adaptación y los atributos de la adaptabilidad que les corresponden, permiten disponer del sistema categorial presentado en la Tabla 1.

Tabla 1.

Capacidad de adaptación y adaptabilidad. Definición de sus rasgos y atributos

\begin{tabular}{|c|c|c|}
\hline \multirow{2}{*}{$\begin{array}{l}\text { CACIDAD DE } \\
\text { ADAPTACIÓN } \\
\text { RASGOS }\end{array}$} & \multicolumn{2}{|r|}{ ADAPTABILIDAD } \\
\hline & ATRIBUTOS & DEFINICIÓN \\
\hline \multirow{4}{*}{\begin{tabular}{l}
\multicolumn{1}{c}{ Criterios } \\
Plataforma de disposición \\
para las acciones. \\
Toma de conciencia de lo \\
demandado para el \\
propio desarrollo \\
profesional docente.
\end{tabular}} & Motivación & $\begin{array}{l}\text { Conducta encauzada a satisfacer necesidades. Se expresa por } \\
\text { la tendencia a auto-realizarse como profesional. }\end{array}$ \\
\hline & Perspicacia & $\begin{array}{l}\text { Capacidad de recabar informaciones para el desarrollo } \\
\text { profesional. Se expresa por la experimentación de actividades. }\end{array}$ \\
\hline & Compromiso & $\begin{array}{l}\text { Disposición a crear las condiciones de realización de acciones } \\
\text { con efecto personal e institucional. Se expresa por la } \\
\text { reiteración de esas acciones. }\end{array}$ \\
\hline & $\begin{array}{l}\text { Condiciones } \\
\text { formativas }\end{array}$ & $\begin{array}{l}\text { Movilización de medios para mejorar la calidad de funciones y } \\
\text { tareas profesionales. Se expresa por la demanda de } \\
\text { orientación y acompañamiento. }\end{array}$ \\
\hline \multirow{3}{*}{$\begin{array}{l}\quad \text { Mecanismos } \\
\text { Plataforma de evolución, } \\
\text { regida por medios } \\
\text { prácticos. } \\
\text { Definición y gestión del } \\
\text { propio desarrollo } \\
\text { profesional. }\end{array}$} & $\begin{array}{l}\text { Apertura al desarrollo } \\
\text { profesional }\end{array}$ & $\begin{array}{l}\text { Inclinación hacia el aprendizaje y experiencias nuevas. Se } \\
\text { expresa por el cuestionamiento, la movilidad y colaboración } \\
\text { con otros. }\end{array}$ \\
\hline & Deseo de progreso & $\begin{array}{l}\text { Gusto por mejorar el propio desarrollo profesional. Se expresa } \\
\text { por la organización de su formación y la satisfacción al } \\
\text { respecto. }\end{array}$ \\
\hline & Determinación & $\begin{array}{l}\text { Impulso para el emprendimiento de acciones. Se expresa por } \\
\text { la firmeza y autonomía en la toma de decisiones individuales e } \\
\text { institucionales. }\end{array}$ \\
\hline
\end{tabular}




\begin{tabular}{|c|c|c|}
\hline & Toma de conciencia & $\begin{array}{l}\text { Sensibilización hacia la propia gestión de la vida profesional. } \\
\text { Se expresa por la voluntad para remediar las necesidades } \\
\text { percibidas. }\end{array}$ \\
\hline \multirow{5}{*}{$\begin{array}{l}\quad \text { Fuerzas } \\
\text { Plataforma de regulación } \\
\text { por la que se aplica el } \\
\text { concepto propio de } \\
\text { profesión. } \\
\text { Rendimiento de la gestión } \\
\text { del propio desarrollo } \\
\text { profesional. }\end{array}$} & Control & $\begin{array}{l}\text { Regulación del desarrollo profesional docente. Se expresa por } \\
\text { la asunción de responsabilidad en la construcción de su } \\
\text { carrera. }\end{array}$ \\
\hline & Participación & $\begin{array}{l}\text { Selección de acciones orientadas al desarrollo profesional. Se } \\
\text { expresa por la planificación de futuro y el emprendimiento de } \\
\text { acciones específicas. }\end{array}$ \\
\hline & Confianza & $\begin{array}{l}\text { Sentimiento para afrontar desafíos de la profesión docente. Se } \\
\text { expresa por el valor y las expectativas de eficacia. }\end{array}$ \\
\hline & Curiosidad & $\begin{array}{l}\text { Inquietud por desempeñar las funciones y tareas en las } \\
\text { mejores condiciones. Se expresa por la preocupación de } \\
\text { mejora y la autocensura. }\end{array}$ \\
\hline & Seguridad & $\begin{array}{l}\text { Ausencia de prevenciones y autosuficiencia. Se expresa por la } \\
\text { certeza en la preparación del desempeño profesional docente. }\end{array}$ \\
\hline
\end{tabular}

Fuente: Elaboración propia, desde fuentes recogidas en Benedicto Carrillo (2016)

A estas definiciones subyacen componentes que integran el sistema comportamental y de la personalidad y sustentan el desarrollo profesional docente continuo. Si bien la capacidad de adaptación y la adaptabilidad son difícilmente medibles, sí pueden ser objeto de observación, análisis y valoración en informaciones aportadas por los docentes sobre lo vivido-experiencial. Desde esta idea, los valores adaptativos de los docentes pueden ser reveladores de su desarrollo profesional a lo largo de la carrera docente. Así pues, son objeto de estudio los indicadores de la adaptación, con la finalidad de profundizar en su valor competencial, aportando conocimiento para las mejores estrategias de desarrollo profesional docente, reconocimiento y cualificación.

\section{Método}

El alcance de la problemática y del objeto de estudio nos lleva a adoptar un enfoque descriptivo e interpretativo en la tradición avalada en Ciencias Sociales y, en este caso, en el ámbito científico que se ocupa de la formación docente. Para una indagación fecunda de la adaptación, se adopta el paradigma cualitativo (Yin, 2016).

La toma de decisiones metodológicas corresponde a los siguientes objetivos:

Objetivo general

- Dar cuenta de la potencialidad de la adaptación como factor competencial clave del perfil profesional de especialistas de la enseñanza bilingüe, con vistas a la mejora de su desarrollo profesional docente continuo.

Objetivos específicos

- Identificar el estado de los rasgos de la capacidad de adaptación y de los atributos de la adaptabilidad en una muestra significativa de docentes en activo.

- Aportar pautas y orientaciones que faciliten atender a la configuración competencial de la adaptación, en la vía de remediar carencias en el desarrollo profesional de este colectivo docente.

\subsection{Participantes}

Para lograr datos productivos, recurrimos a las posibilidades que ofrecen el muestreo opinático (Abreu Abela, 2002) y el acceso a una muestra por contraste-profundización (Pirès, 1997), de carácter intencional e invitada (Patton, 1990). Los criterios de selección están presididos por el de la heterogeneidad máxima buscada respecto de las etapas de desarrollo profesional ya descritas, y de las situaciones docentes contractuales. Se añadieron además los criterios de disponibilidad-voluntariedad y compromiso requeridos para disponer del mayor número posible de informaciones. Resulta una muestra final con las siguientes características:

- Localizada en el contexto escolar castellanoleonés, al estar marcado por un continuo incremento de programas AICLE en las secciones bilingües, y el elevado contingente de alumnos beneficiarios. Asimismo, por la facilidad de acceso y contactos por parte de los investigadores. 
- Compuesta por cinco Informantes $(\ln 1, \ln 2, \ln 3, \ln 4, \ln 5)$, docentes especialistas de la enseñanza bilingüe correspondientes a las cinco etapas de desarrollo profesional, y en situaciones docentes contractuales diversas.

En la Tabla 2 se describe su configuración:

Tabla 2.

Muestra de Informantes distribuidos por etapas de desarrollo profesional y situaciones docentes

\begin{tabular}{|c|c|c|}
\hline $\begin{array}{l}\text { Sujetos } \\
\text { Informantes }\end{array}$ & $\begin{array}{l}\text { Etapa de desarrollo } \\
\text { profesional }\end{array}$ & Situaciones docentes contractuales \\
\hline Informante 1 & Transición & $\begin{array}{l}\text {-Docente de inglés y de contenidos curriculares en inglés en } \\
\text { Educación Primaria } \\
\text {-Centro concertado }\end{array}$ \\
\hline Informante 2 & Preparación a la jubilación & $\begin{array}{l}\text {-Docente de inglés y de contenidos curriculares en inglés en } \\
\text { Educación Primaria } \\
\text {-Centro público }\end{array}$ \\
\hline Informante 3 & Crisis de la carrera & $\begin{array}{l}\text {-Docente de inglés y de contenidos curriculares en inglés en } \\
\text { Educación Primaria } \\
\text {-Centro público }\end{array}$ \\
\hline Informante 4 & Ingreso a la profesión & $\begin{array}{l}\text {-Docente de inglés y de contenidos curriculares en inglés en } \\
\text { Educación Secundaria Obligatoria } \\
\text {-Centro concertado }\end{array}$ \\
\hline Informante 5 & Estabilización y compromiso & $\begin{array}{l}\text {-Docente de contenidos curriculares en inglés en Educación } \\
\text { Secundaria Obligatoria y Bachillerato } \\
\text {-Centro público }\end{array}$ \\
\hline
\end{tabular}

\subsection{Instrumentos de recogida de datos}

Para obtener un corpus de datos contextualizados sobre los rasgos de la capacidad de adaptación y los atributos de la adaptabilidad, se utilizaron el cuestionario y el relato de vida (Burrick, 2010) de forma individual y complementaria. Por una parte, para consignar informaciones precisas respecto al desarrollo profesional, dado el carácter más controlado del cuestionario; y por otra, para evocar la memoria episódica sobre lo vividoexperiencial, dado el carácter testimonial que encierra el relato de vida.

La administración del cuestionario y la solicitud del relato de vida a cada Informante, se realizaron tras las fases de su concepción, elaboración y validación por pares, para asegurar:

- Que el cuestionario presentase un alto grado de operatividad. Se necesitaba disponer del mayor número posible de respuestas, ante la variada tipología de ítems (respuestas abiertas, cerradas, etc.) necesaria para abarcar el amplio espectro del sistema comportamental y del de la personalidad. Con estos requisitos, el cuestionario está configurado para obtener: a) Biodata (siete ítems); b) Determinaciones socio-académicas (ocho ítems); c) Determinaciones socio-profesionales (tres ítems). (Ver en Anexo).

- Que el relato de vida apelase al yo hermenéutico. Se necesitaba una escritura reflexiva, autobiográfica y temporal de la trayectoria profesional con carácter retrospectivo (Sanz Trigueros y Guillén Díaz, 2019). Con estos requisitos, se articula la consigna en tres enunciados sobre: experiencia profesional; autopercepciones; cualificaciones y formación continua.

\subsection{Procedimiento}

Para la investigación cualitativa-interpretativa, la flexibilidad del análisis de contenido (Bardin, 2002) hace pertinente su empleo como técnica global por excelencia. Para ello:

- El sistema categorial de referencia está presidido por la macro-categoría adaptación, y se establece con los elementos definidos en la Tabla 1. Son categorías de análisis los rasgos de la capacidad de adaptación; y son subcategorías los atributos de la adaptabilidad.

- Las unidades de contexto son el cuestionario $(\mathrm{Cu})$ y el relato de vida $(\mathrm{Re})$, las unidades de análisis son sus partes ya descritas, y las unidades de registro son los segmentos de información con indicadores de los rasgos de la capacidad de adaptación y de los atributos de la adaptabilidad. 
- El tratamiento de las informaciones es manual (Wanlin, 2007), dada la complejidad organizativa de los datos, ya que sirven a la imbricación entre: a) los niveles de la reflexividad descriptivo, narrativo y valorativo (Van Manen, 1977), asociados con los modos discursivos descripción, narración y valoración; y b) las dimensiones contextual, declarativa, procedimental, intencional y evaluativa, de las informaciones satélites de las acciones orientadas al desarrollo profesional (Vermersch, 2012).

- Las unidades de registro se acompañan de un sistema de códigos alfabético, numérico y cromático que facilita el análisis e interpretación de los resultados.

- La intensidad, parcial y total, es la regla de recuento de los datos registrados para una presentación ágil y verosímil de los valores de los resultados (Bardin, 2002).

- La fiabilidad se asegura desde los criterios de precisión y verdad, aplicados por tres expertos.

Finalmente, se elaboró un dispositivo, utilizable como soporte de análisis por cada a) Nivel de la reflexividadModo discursivo; b) Dimensión de la información; y c) Informante, tal y como ilustramos en la Tabla 3.

\section{Tabla 3}

Dispositivo del análisis de contenido por cada: a) Nivel de la reflexividad - Modo discursivo; b) Dimensión de la información; $y$ c) Informante

\begin{tabular}{|c|c|c|}
\hline \multicolumn{3}{|c|}{ Nivel-Modo (Descriptivo/Narrativo/Valorativo) } \\
\hline Dimensión C & ual/Declarativa/Procedimental//ntencional/Evaluativa & \\
\hline \multicolumn{3}{|c|}{ Informante ... } \\
\hline \multicolumn{2}{|c|}{ Categorías y subcategorías de análisis } & Intensidad Total \\
\hline \multirow{4}{*}{ Criterios } & Motivación & $\ldots$ \\
\hline & Perspicacia & \\
\hline & Compromiso & \\
\hline & Condiciones formativas & \\
\hline \multirow{4}{*}{ Mecanismos } & Apertura al desarrollo profesional & \\
\hline & Deseo de progreso & \\
\hline & Determinación & \\
\hline & Toma de conciencia & \\
\hline \multirow{5}{*}{ Fuerzas } & Control & \\
\hline & Participación & \\
\hline & Confianza & \\
\hline & Curiosidad & \\
\hline & Seguridad & \\
\hline
\end{tabular}

El análisis se efectúa mediante una secuencia de lecturas:

- Lectura 1. Intuitiva, correspondiente a un análisis de contenido estructural y organizativo; preparación de las informaciones.

- Lectura 2. Exploratoria, correspondiente a un análisis de contenido temático; identificación, registro y reducción de datos.

- Lectura 3. Interpretativo-inferencial, correspondiente a un análisis de contenido relacional; de interpretación de resultados.

\section{Resultados}

Los resultados obtenidos se presentan en torno a los rasgos de la capacidad de adaptación y a los atributos de la adaptabilidad.

En cuanto a los rasgos de la capacidad de adaptación, el panorama global de los valores adaptativos se muestra en el Gráfico 1: 


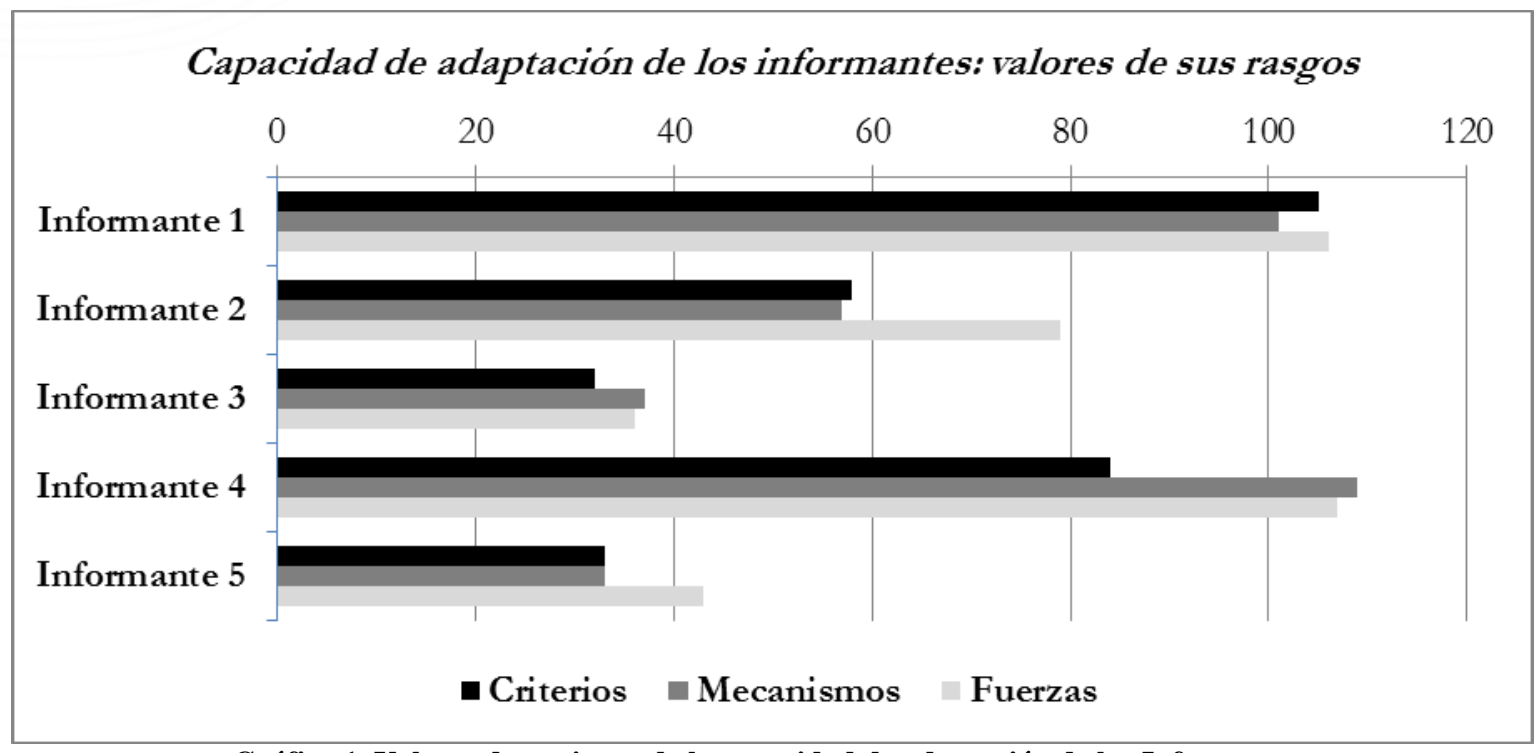

Gráfico 1. Valores de conjunto de la capacidad de adaptación de los Informantes

Estos valores indican un bajo potencial estructural de la capacidad de adaptación de los Informantes, puesto que:

a) Solo el rasgo Fuerzas (Rendimiento de la gestión del propio desarrollo profesional) presenta valores superiores a los demás rasgos. Esto puede verse en los siguientes ejemplos: "Continuar en la misma línea, seguir participando en actividades de formación y/o reciclaje..., no abandonar las ganas por enseñar..." (Cu14-In4); “...el afán innovador que esté presente siempre..." (Re14-In1).

b) Los valores inferiores de los Informantes se encuentran con indicadores carenciales en Criterios (Toma de conciencia de lo que se demanda para el propio desarrollo profesional docente) y en Mecanismos (Definición y gestión del propio desarrollo profesional). Esto puede verse, respectivamente, en los siguientes ejemplos: "...el motivo del cambio ha sido aproximarme al domicilio familiar..." (Re6-In3); “...me considero creativo...Me aburro con rapidez, necesito novedad y cambios" (Re39-In3).

c) Todos los rasgos presentan valores inferiores en los Informantes 3 y 5 , dando cuenta de un desarrollo profesional más propio de la etapa de Transición, aunque pertenecen respectivamente a las etapas de Crisis de la carrera y Estabilización y compromiso.

d) Los valores superiores en todos los rasgos corresponden a los Informantes 1 y 4 , revelando un desarrollo profesional propio de la etapa de Estabilización y compromiso, aunque pertenecen respectivamente a las etapas de Transición y de Ingreso en la profesión.

En cuanto a los atributos de la adaptabilidad, el panorama global de los valores adaptativos se muestra en el Gráfico 2: 


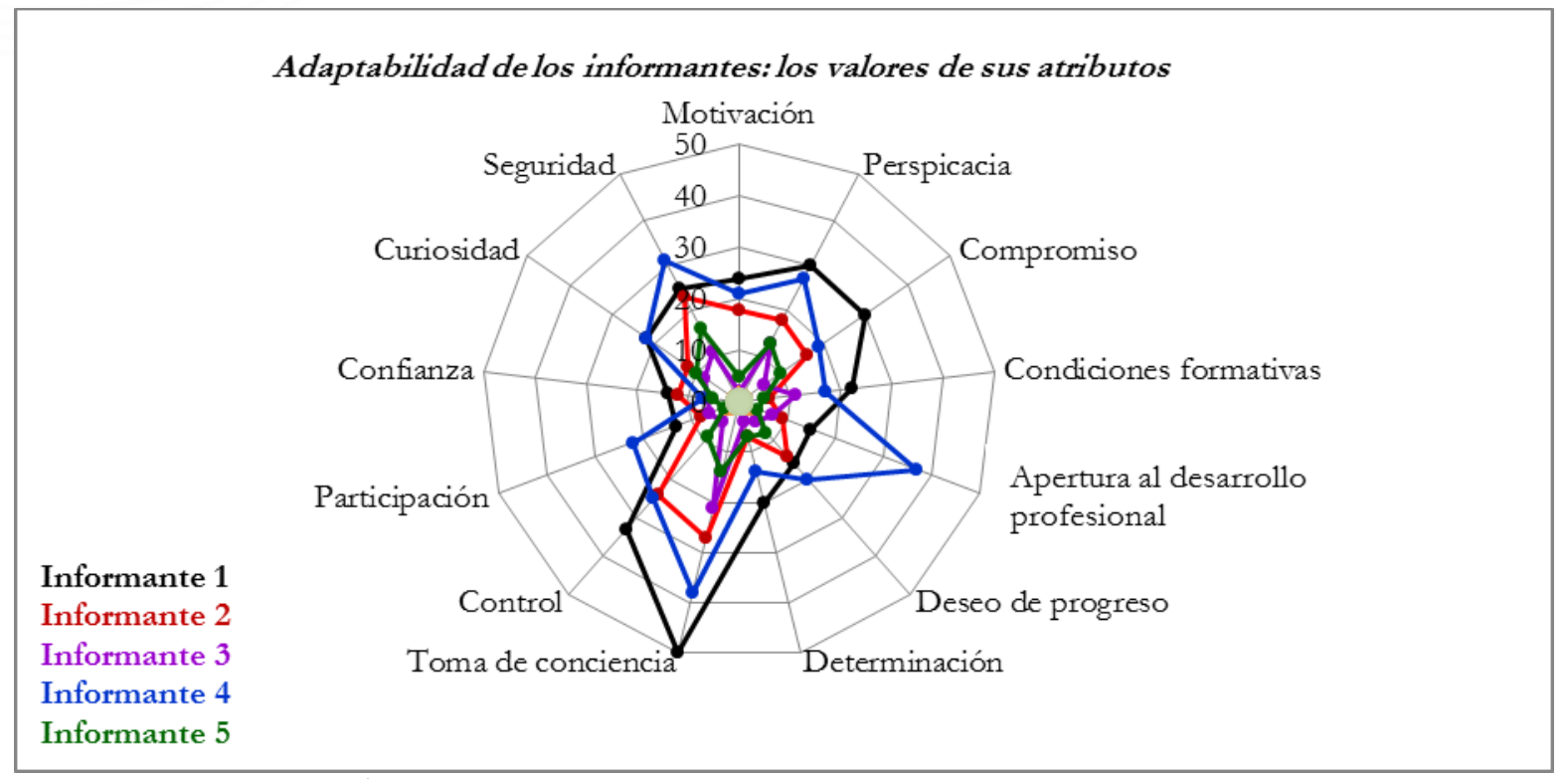

Gráfico 2. Valores de conjunto de la adaptabilidad de los Informantes

Estos valores indican el escaso equilibrio de los atributos de la adaptabilidad de los Informantes. Aun contando con su complementariedad, la presencia de los atributos dominantes Toma de conciencia, Seguridad y Perspicacia convierte a los carenciales Confianza, Participación y Determinación, en no compensatorios.

En los Informantes 3 y 5 se identifican también los atributos carenciales dando cuenta de su desarrollo profesional docente más bien propio de la etapa de Transición, aun perteneciendo respectivamente a las de Crisis de la carrera y de Estabilización y compromiso.

Agrupando los valores de los atributos de cada rasgo, se procede a identificar el estado de la adaptabilidad de los Informantes, y a valorar el estado del sistema comportamental y del de la personalidad de estos.

En el Gráfico 3 se muestran los valores de los atributos correspondientes al rasgo Criterios:

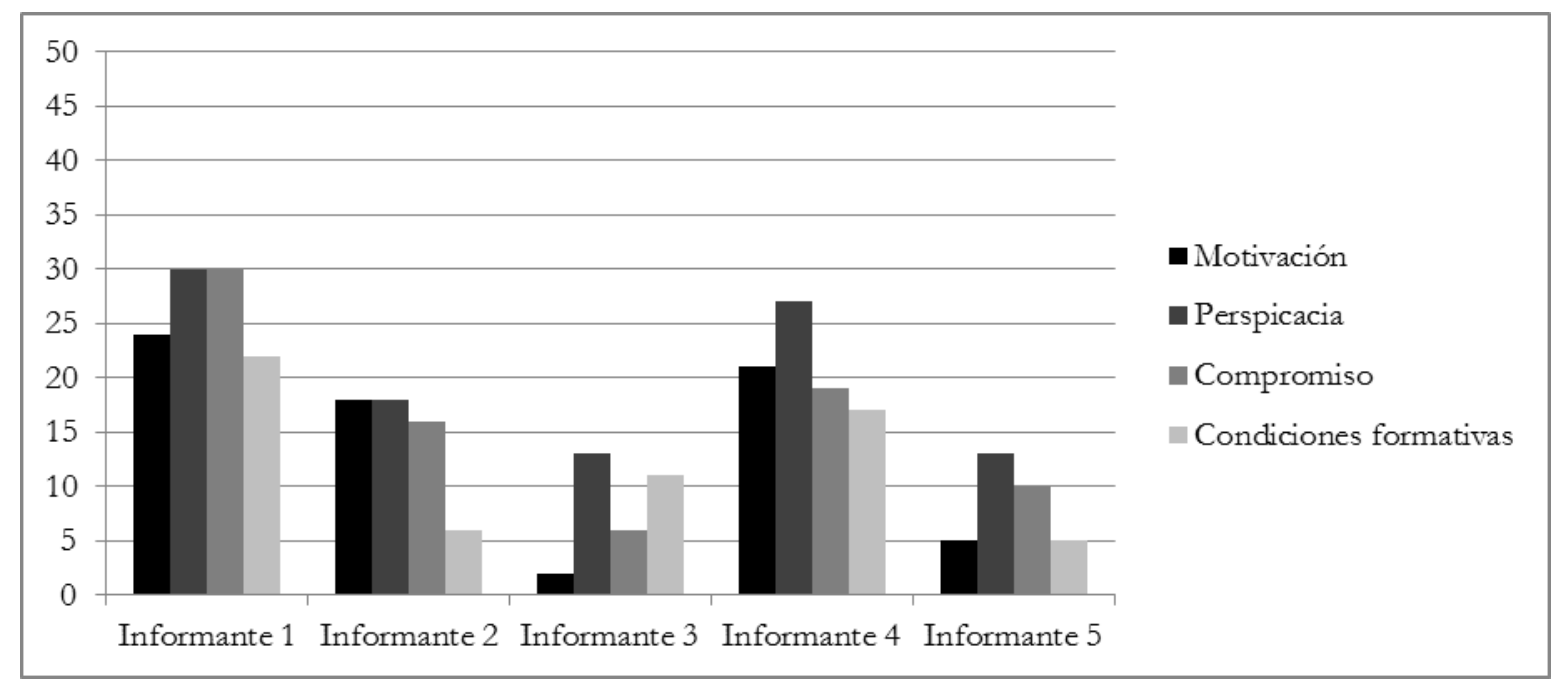

Gráfico 3. Adaptabilidad de los Informantes ante los valores del rasgo Criterios

Cabe interpretar que el estado de sus sistemas comportamental y de la personalidad es poco adecuado. Lo confirma el hecho de no poder intuir la disposición para la adaptabilidad, a pesar de que los valores de la Perspicacia y el Compromiso se revelan, en su complementariedad, compensatorios del resto de los atributos. Esto puede verse en los siguientes ejemplos: "...veo una gran dificultad en impartir clase de idioma extranjero" (Re7-In3); "...cuando ves que tu nivel de inglés resulta insuficiente..., genera mucha inseguridad" (R11-In5); “...voy a necesitar un curso escolar más para sentirme totalmente cómoda y adaptada" (R12-In5). 
En el Gráfico 4, se muestran los valores de los atributos del rasgo Mecanismos:

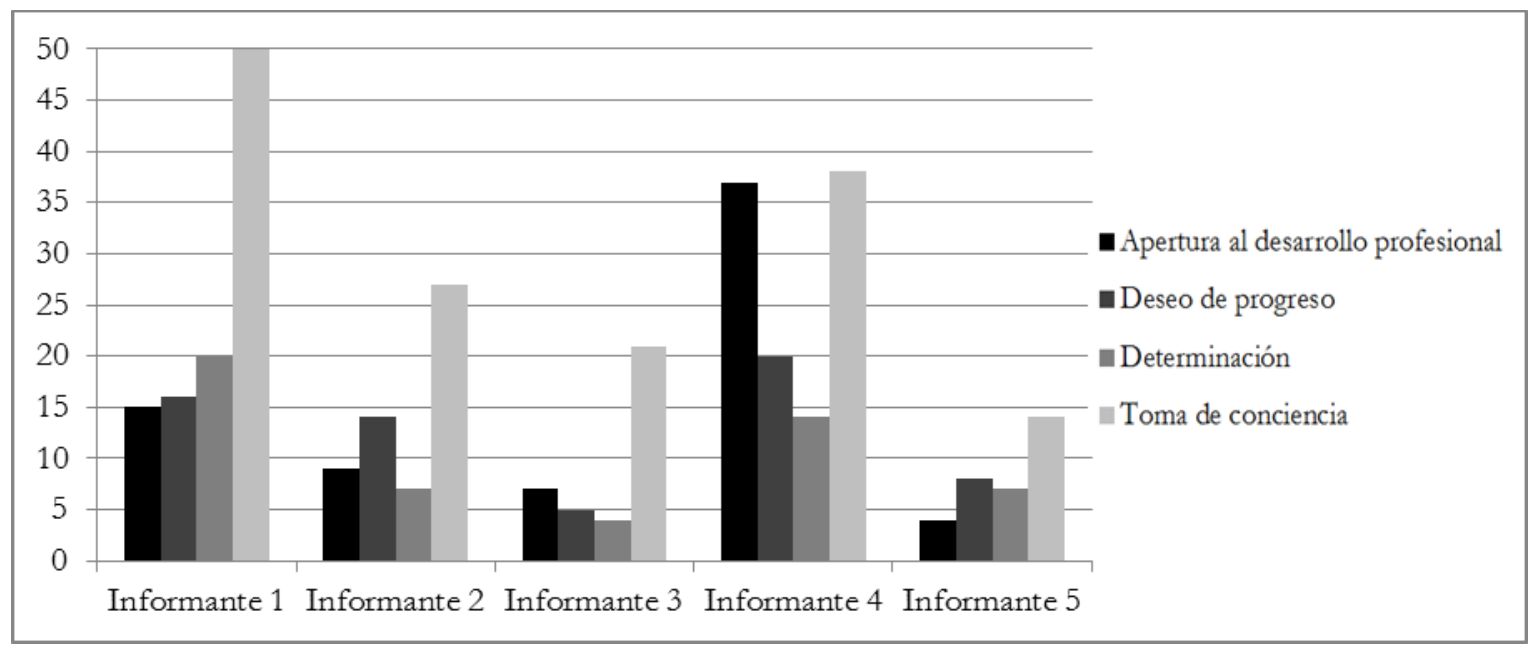

Gráfico 4. Adaptabilidad de los Informantes ante los valores del rasgo Mecanismos

Cabe interpretar que el estado de su sistema comportamental es poco adecuado. Se corrobora por no apreciarse desde los valores de la Determinación -atributo carencial- la evolución hacia la adaptabilidad de estos Informantes, aun reconociendo que el atributo Apertura al desarrollo profesional puede actuar como compensatorio, en particular en el Informante 4. Esto puede verse en los siguientes ejemplos: “...sin intención de cambiar."(Re7-In2); "No me imagino haciendo otra cosa." (Re11-In2). Y, respecto al estado de su sistema de la personalidad se interpreta que es adecuado, ya que el atributo Deseo de progreso permite dar cuenta de la evolución hacia la adaptabilidad de estos Informantes, considerando al mismo tiempo al atributo dominante Toma de conciencia como compensatorio, en particular en el Informante 1. Esto puede verse en los siguientes ejemplos: “...cualquier oportunidad es buena para aprender." (Cu14-In4); "Me gustaría mejorar mi competencia didáctica." (Re20-In4).

Por otro lado, en el Gráfico 5 se muestran los valores de los atributos del rasgo Fuerzas:

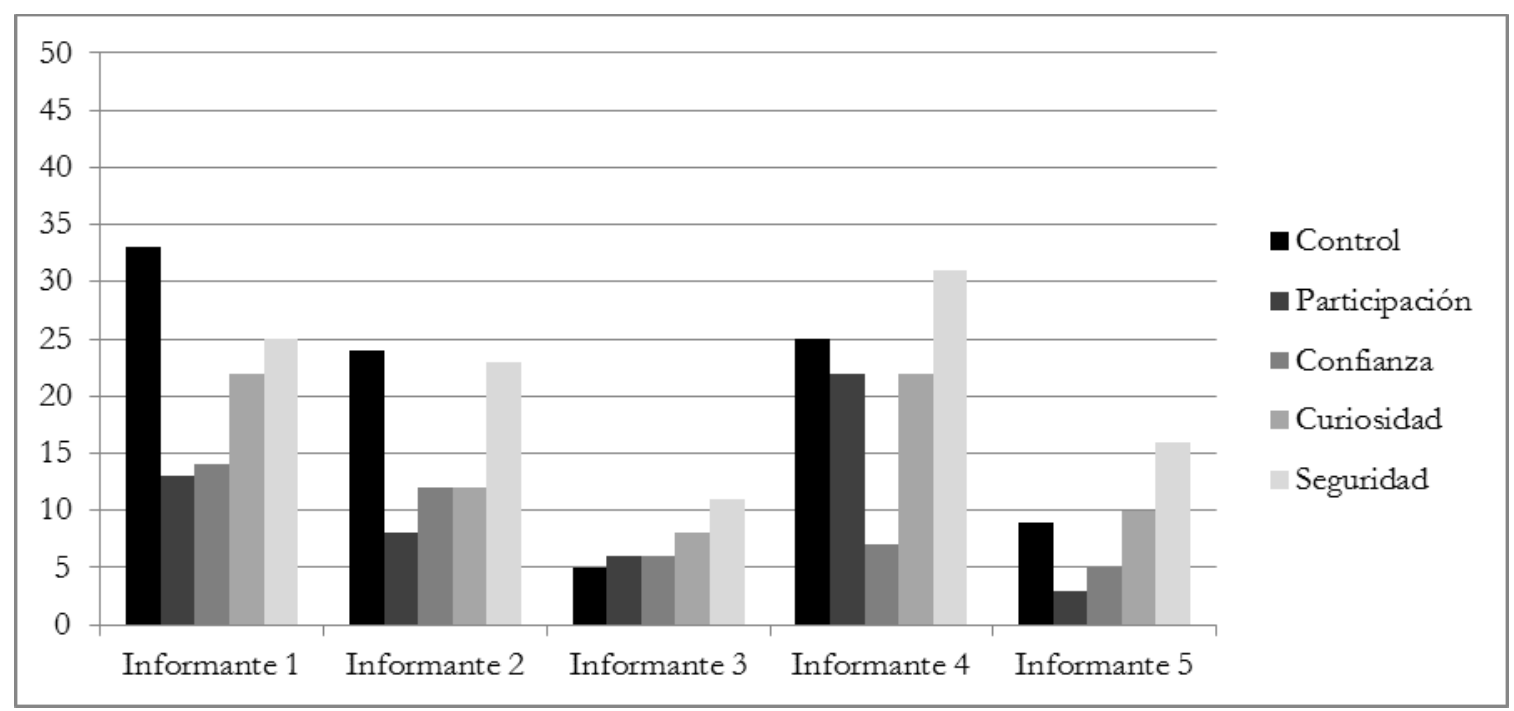

Gráfico 5. Adaptabilidad de los Informantes ante los valores del rasgo Fuerzas

Cabe interpretar que el estado de sus sistemas comportamental y de la personalidad es poco adecuado. Se confirma porque los valores de Participación, Confianza y Curiosidad impiden tener una visión de la regulación de la adaptabilidad, si bien Control y Seguridad revelan su carácter compensatorio. Esto puede verse en los 
siguientes ejemplos: "...hacer una valoración-revisión de cualidades, a falta de ella" (Cu8-In3); "Tengo carencias en el idioma." (R15-In5).

Con todo, parece lógico inferir una baja tendencia hacia la excelencia en el desarrollo profesional de estos docentes, por cuanto el panorama global de la adaptación, en este caso, se caracteriza por un bajo potencial estructural de la capacidad de adaptación, un escaso equilibrio y carácter compensatorio de los atributos de la adaptabilidad, y un estado poco adecuado de sus sistemas comportamental y de la personalidad.

\section{Discusión}

Las características de la adaptación -en todas las etapas de desarrollo profesional descritas, excepto en la de Preparación a la jubilación-, dan cuenta de:

-Escasa tendencia hacia la excelencia del desarrollo profesional como docentes especialistas de la enseñanza bilingüe, en el sentido de European Commission (2019).

-Aspectos susceptibles de reflexión para los formadores de estos docentes e instancias encargadas de su atención, como aportan Parsons et al. (2011) o Vaillant (2014).

Tendencia y aspectos en la línea de López Rupérez (2014) y de estudios exploratorios realizados en diversos contextos sobre las representaciones y percepciones de estos docentes. Entre otros, Travé González (2013) y Steffen (2019) pormenorizando el problema del tratamiento metodológico de la lengua y el contenido de la disciplina no lingüística; y Durán-Martínez, Beltrán-Llavador y Martínez Abad (2016), revelando las diferencias por experiencia de los docentes.

Sin pretender una generalización, la mejora del desarrollo profesional de los docentes implicados en la enseñanza bilingüe puede ser gestionada por los formadores universitarios, focalizando la atención en los rasgos Criterios, Mecanismos y Fuerzas, por constituir estos valiosas plataformas de indagación reveladoras del potencial estructural de la capacidad de adaptación, en las perspectivas psico-socio-cognitiva y profesionalizadora. Por un lado, el rasgo Criterios es necesario porque, desde los atributos Motivación, Perspicacia, Compromiso y Condiciones formativas, se fortalecería el sistema de la personalidad de los especialistas de la enseñanza bilingüe. Por otro lado, se han de entrenar los Mecanismos porque, desde los atributos de Apertura al desarrollo profesional, Deseo de progreso, Determinación y Toma de conciencia, se orientaría su sistema comportamental. Finalmente, es necesario el rasgo Fuerzas porque, desde los atributos Control, Participación, Confianza, Curiosidad y Seguridad, se facilitaría la regulación de ambos sistemas.

Cabe igualmente considerar que los atributos han de ser también un foco de atención importante en la formación. Por un lado, la Toma de conciencia y Control -dominantes en todas las etapas de la carrera observadas- son puntos de partida clave para orientar y acompañar a este colectivo profesional emergente en la optimización de su desarrollo profesional. Por otro lado, la Confianza, Participación y Condiciones formativas -carenciales excepto en el caso de la etapa de Ingreso a la profesión-son un importante foco de atención, por la contradicción que sus valores revelan ante los de la Toma de conciencia. Finalmente, la Motivación, Perspicacia, Compromiso, Deseo de progreso y Determinación, junto a Curiosidad y Seguridad, si bien no presentan valores realmente carenciales, sí han de ser considerados importantes, pues facilitarían la regulación y adecuación de los sistemas comportamental y de la personalidad de estos docentes.

De estas "zonas de sombra" (Hébrard, 2011) -por carencias principalmente- en la adaptabilidad se desprende que el desarrollo profesional continuo es un deber prioritario (Bokdam, Van den En y Broek, 2014), y un derecho, en la perspectiva del reconocimiento de las cualificaciones docentes.

\section{Conclusiones}

Conforme a las exigencias de las políticas sectoriales (lingüística, educativa, de empleo y de calidad) que vienen marcando el ámbito socioeducativo actual, cabe concluir que el sector profesional de especialistas de la enseñanza bilingüe debe y debe poder, por un lado, ampliar las opciones de empleabilidad ante el incremento exponencial de secciones bilingües, y promocionarse profesionalmente desde la plena proyección de este componente competencial actual clave que es la adaptación, asegurando la adecuación y correspondencia entre etapas de desarrollo profesional y el estado del desarrollo profesional.

Por otro lado, gestionar su proyecto de desarrollo profesional continuo, atendido por formadores universitarios, quienes utilizarían estrategias de confrontación al estado de la capacidad de adaptación y la adaptabilidad, institucionalizando ya desde la formación inicial la aplicación de dispositivos y medios de orientación y acompañamiento. 
En este orden de cosas, cabe plantear propuestas orientadas a aumentar las expectativas necesarias para planificar y gestionar el desarrollo profesional a lo largo de la carrera del docente implicado en la enseñanza bilingüe. Se trata, tomando como plataforma de referencia la adaptación, de proveer de:

- Figuras de orientación, acompañamiento-seguimiento experto (mentoría, tutorización, etc.) en la toma de decisiones y previsión del itinerario profesional docente que mejor se corresponda, como apoyo a los atributos Motivación, Compromiso y Determinación.

- Amparo institucional que se ocupe de vigilar la actualización del proyecto personal de desarrollo profesional docente continuo, facilitando un progreso sostenible de las funciones y tareas profesionales a las que la enseñanza bilingüe confronta al docente especialista.

- Recursos y medios suficientes para garantizar una estrategia de profesionalización coherente, en la perspectiva del perfeccionamiento continuo que se reclama desde estudios internacionales (Bokdam, Van den En y Broek, 2014; Vaillant, 2014).

- Condiciones adecuadas para la aplicación de una auditoría de competencias que, tomada en la lógica de la Recomendación 2012/C 398/01 del Consejo de la Unión Europea sobre la validación del aprendizaje no formal e informal (Unión Europea, 2012), fortalezca el reconocimiento social e institucional del perfil profesional docente de este colectivo.

- Procedimientos institucionales de regulación de las cualificaciones profesionales como docentes especialistas, contemplando la validación de sus aprendizajes adquiridos por la experiencia, con vistas a hacer efectivas las oportunidades de empleabilidad.

El alcance de estos resultados se complementaría por trabajos (aún insuficientes) que, en los escenarios complejos (Morin, 2015) de los proyectos bilingües, aborden las interacciones significativas entre tres variables básicas, complejas a su vez: el alumnado, los recursos (humanos y materiales), y el objeto (programa AICLE del que se trate).

\section{Referencias}

Abreu Abela, J. (2002). Las técnicas de análisis de contenido: Una revisión actualizada. Recuperado el 23 de febrero de 2020 de https://bit.ly/2RoPO5Q

Bardin, L. (2002). Análisis de contenido. Madrid: Akal.

Bauman, Z. (2007). Los retos de la educación en la modernidad líquida. Barcelona: Gedisa.

Beacco, J. C., Byram, M., Cavalli, M., Coste, D., Egli Cuenat, M., Goullier, F. y Panthier, J. (2016). Guide for the development and implementation of curricula for plurilingual and intercultural education. Estrasburgo: Council of Europe. Recuperado el 27 de febrero de 2020 de https://bit.ly/2RmsS71

Benedicto Carrillo, E.C. (2016). Toma de decisiones en sistemas dinámicos no lineales. Una perspectiva psicosociológica (tesis doctoral). Universidad de Valladolid. https://doi 10.35376/10324/16403

Bertalanffy, L. von (1986). Teoría general de los sistemas. Fundamentos, desarrollo y aplicaciones. Madrid: Fondo de Cultura Económica.

Bleichenbacher, L. (Coord.) (2019). Teacher competences for languages in education. Conclusions of the project. Graz: European Centre for Modern Languages y Council of Europe. Recuperado el 4 de marzo de 2020 de https://bit.ly/2USZ2JN Bokdam, J., Van den En, I. y Broek, S. (2014). Teaching teachers: primary teacher training in Europe - state of affairs and outlook study. Estrasburgo: European Parliament. Recuperado el 13 de enero de 2020 de https://bit.ly/2USZesv

Burrick, D. (2010). Une épistémologie du récit de vie. Recherches Qualitatives -Hors Série, 8, 7-36.

Comisión Europea, EACEA y Eurydice (2018). La profesión docente en Europa. Acceso, progresión y apoyo. Informe de Eurydice. Luxemburgo: Oficina de Publicaciones de la Unión Europea. Recuperado el 25 de enero de 2020 de https://bit.ly/3c2jOHs

Coyle, D., Hood, P. y Marsh, D. (2010). CLIL: Content and Language Integrated Learning. Nueva York: Cambridge University Press.

Daft, R. L. (2007). Teoría y diseño organizacional. México: Thomson.

Durán-Martínez, R., Beltrán-Llavador, F. y Martínez Abad, F. (2016). A contrastive analysis between novice and expert teachers' perceptions of school bilingual programmes. Culture and Education, 28(4), 738-770. https://doi.org/10.1080/11356405.2016.1237339

EPyCE (2019). Posiciones y competencias más demandadas. Barcelona y Madrid: EAE Business School y Asociación Española de Directores de Recursos Humanos. Recuperado el 2 de marzo de 2020 de https://bit.ly/2JLVL8A

Escobar Urmeneta, C. (2019). An Introduction to Content and Language Integrated Learning (CLIL) for teachers and teacher educators. CLIL Journal of Innovation and Research in Plurilingual and Pluricultural Education, 2(1), 7-19. https://doi.org/10.5565/rev/clil.21

European Commission (2015a). The knowledge future: intelligent policy choices for Europe 2050. A report to the European Commission. Bruselas: European Commission. Recuperado el 9 de febrero de 2020 de https://bit.ly/3e3EbLh

European Commission (2015b). The teaching profession in Europe: Practices, perceptions, and policies. Luxemburgo: Publications Office of the European Union. Recuperado el 18 de febrero de $2020 \mathrm{de} \mathrm{https://bit.ly/2VeOLpV}$ 
European Commission (2019). Innovating professional development in compulsory education. An analysis of practices aimed at improving teaching and learning. Luxemburgo: Publications Office of the European Union. https://doi.org/10.2760/948518

Fernández Aráoz, C. (2008). La toma de decisiones sobre capital humano en el nuevo entorno global. Harvard Deusto Business Review, 165, 42-48. Recuperado el 23 de marzo de https://bit.ly/2VaWZ2y

González Piñeiro, M., Guillén Díaz, C. y Vez Jeremías, J. M. (2010). Didáctica de las lenguas modernas: competencia plurilingüe e intercultural. Madrid: Síntesis.

Hébrard, P. (2011). L'humanité comme compétence? Une 'zone d'ombre' dans la professionnalisation aux métiers de l'interaction avec autrui. Les Sciences de l'Éducation - Pour l'Ére Nouvelle, 44(2), 103-121. https://doi.org/10.3917//sdle.442.0103

Hirschi, A. (2015). Career adaptability profiles and their relationship to adaptivity and adapting. Journal of Vocational Behavior, 88, 220-229. https://doi.org/10.1016/j.jvb.2015.03.010

Kiely, R. (2011). Understanding CLIL as an innovation. Studies in Second Language Learning and Teaching, 1(1), 153171. https://doi.org/10.14746/ssllt.2011.1.1.9

López Rupérez, F. (2014). Fortalecer la profesión docente. Un desafío crucial. Madrid: Narcea.

Morin, E. (2015). Penser global. L'humain et son univers. Paris: Robert Laffont. Recuperado el 16 de febrero de 2020 de https://bit.ly/3bVfbns

Parsons, S. A., Massey, D., Vaughn, M., Scales, R. Q., Faircloth, B. S., Howerton, W. y Atkinson, T. S. (2011). Developing teachers' reflective thinking and adaptability in graduate courses. Journal of School Connections, 1(3), 91-111.

Patton, M. Q. (1990). Qualitative evaluation and research methods. Londres: Sage. https://doi.org/10.1002/nur.4770140111

Pérez-Cañado, M. L. (2018) Innovation and Challenges in CLIL teacher education. Theory Into Practice, 57(3), 1-10. https://doi.org/10.1080/00405841.2018.1492238

Pérez Gómez, A., Barquín Ruiz, J. y Angulo Rasco, J. F. (Eds.) (1999). Desarrollo profesional del docente. Política, investigación y práctica. Madrid: Akal. https://doi.org/10.18172/con.418

Pirès, A. P. (1997). Échantillonnage et recherche qualitative: essai théorique et méthodologique. En J. Poupart, J. P. Deslauriers, L. H. Groulx, R. Mayer y A. Pirès, La recherche qualitative: Enjeux épistémologiques et méthodologiques (pp. 113-167). Montreal: Gaëtan Morin.

Puren, C. (1998). Concepts et conceptualisation en Didactique des Langues: Pour une épistémologie disciplinaire. Didáctica (Lengua y Literatura), 10, 271-288.

Sanz Trigueros, F. J. (2018). Desarrollo profesional y adaptabilidad del docente de y en lenguas extranjeras en las dinámicas europeas. Un estudio para sus cualificaciones en la enseñanza obligatoria (tesis doctoral). Universidad de Valladolid. Recuperado de https://bit.ly/3fChSfj

Sanz Trigueros, F. J. y Guillén Díaz, C. (2019). El relato de vida en la inter y transdisciplinariedad de la investigación en didáctica de la lengua y la literatura. En Mạ . C. Quiles Cabrera y M. Campos Fernández-Fígares, Repensando la didáctica de la lengua y la literatura. Paradigmas y líneas emergentes de investigación (pp. 429-442). Madrid: Visor Libros.

Savickas, M. L. (2005). The theory and practice of career construction. En S. D. Brown y R. W. Lent (Eds.), Career development and counseling: Putting theory and research to work (pp. 42-70). Hoboken, New Jersey: Wiley.

Simonet, G. (2009). Le concept d'adaptation: Polysémie interdisciplinaire et implication pour les changements climatiques. Revue Natures Sciences Société, 4(17), 392-401. https://doi.org/10.1051/nss/2009061

Steffen, G. (2019). L'enseignement bilingue au croisement de différentes approches didactiques: perspectives des enseignants. Revue TDFLE, 74. Recuperado el 3 de abril de 2020 de https://bit.ly/2XuSp0R

Super, D. E. (1980). A life-span, life-space approach to career development. Journal of Vocational Behavior, 16(3), 282298. https://doi.org/10.1016/0001-8791(80)90056-1

Travé González, G. (2013). Un estudio sobre las representaciones del profesorado de Educación Primaria acerca de la enseñanza bilingüe. Revista de Educación, 361, 379-402. https://doi.org/10.4438/1988-592X-RE-2011-361-149

Unión Europea (2012). Recomendación del Consejo de 20 de diciembre de 2012 sobre la validación del aprendizaje no formal e informal (2012/C 398/01). Diario oficial de la Unión Europea (22/12/2012). Recuperado el 29 de marzo de 2020 de https://bit.ly/3aUODEG

Vaillant, D. (2014). Análisis y reflexiones para pensar el desarrollo profesional docente continuo. Educar, 50, 55-66. https://doi.org/10.5565/rev/educar.690

Van Manen, J. (1977). Linking ways of knowing with ways of being practical. Curriculum Inquiry, 6, $205-228$. https://doi.org/10.1080/03626784.1977.11075533

Vermersch, P. (2012). Explicitation et phénoménologie. París: Presses Universitaires de France.

Wanlin, P. (2007). L'analyse de contenu comme méthode d'analyse qualitative d'entretiens: une comparaison entre les traitements manuels et l'utilisation des logiciels. Recherches Qualitatives -Hors Série, 3, 243-272.

Yin, R. K. (2016). Qualitative research from start to finish. Londres: The Guilford Press. 
Anexo. Formato Síntesis del Cuestionario administrado

En los siguientes apartados, marque las casillas que corresponden a su situación actual y/o escriba la información solicitada
1- Edad: Menos de $30 \square$
$30-40$
$40-50$
2- Sexo: Mujer
Hombre
3- Nacionalidad
4- Lengua materna
Lengua(s) que domina o ha estudiado
5- Titulación de origen
Últimos estudios
6- Desempeña su labor profesional en:
$\square$ Primaria profesor/a de
$\square$ Secundaria
Bachillerato
Otro contexto
profesor/a de
profesor/a de
profesor/a de
profesor/a de
años
— años
— años

50-65

7- Otros puestos de trabajo y períodos:

8- Ante los ámbitos de formación continua para el profesorado en Lenguas Extranjeras, indique su participación

\begin{tabular}{|l|l|l|}
\hline Título, Orientación temática \\
\hline № de horas & 10 & 20 \\
\hline
\end{tabular}

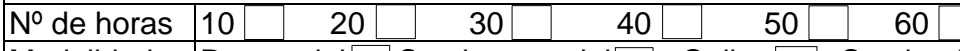

\begin{tabular}{|l|lrrrr} 
& & & \\
\hline Modalidad & Presencial $\square$ & Semipresencial $\square$ & Online $\square$ & Seminario/jornada $\square$ & Grupo de trabajo $\square$ \\
\hline Tipología & Estancias $\square \square$ & Congreso $\square$ & *MOOC & Curso $\square$ & Formación en Red $\square$ \\
\hline
\end{tabular}

$\checkmark$ Utilice cuantas casillas necesite en el reverso

9- Del siguiente listado de competencias:

a) Valore la importancia de cada una y el nivel que considera logrado, conforme a estas escalas

\begin{tabular}{|c|c|c|c|c|c|c|c|c|}
\hline \multirow{2}{*}{\begin{tabular}{|c|}
\multicolumn{1}{|c|}{ Competencias } \\
(cognitivas, funcionales, personales y sociales) \\
\end{tabular}} & \multicolumn{4}{|c|}{\begin{tabular}{|l|} 
Atribución de importancia \\
\end{tabular}} & \multicolumn{4}{|c|}{ Nivel de logro } \\
\hline & mucho & bastante & algo & poco & & 2 & & 4 \\
\hline \multicolumn{9}{|l|}{ Conocimiento en Educación } \\
\hline \multicolumn{9}{|l|}{ Gestión del conocimiento } \\
\hline \multicolumn{9}{|l|}{ Normativa y programación } \\
\hline \multicolumn{9}{|l|}{ Metodología, actividades y evaluación } \\
\hline \multicolumn{9}{|l|}{ Gestión del aula, recursos y materiales } \\
\hline \multirow{2}{*}{\multicolumn{9}{|c|}{ Organización, planificación, coordinación }} \\
\hline & & & & & & & & Gestión de calidad \\
\hline \multicolumn{9}{|l|}{ Promoción de la convivencia } \\
\hline \multicolumn{9}{|l|}{ Actitudes de cooperación y colaboración } \\
\hline \multicolumn{9}{|l|}{ Participación en Proyectos comunes } \\
\hline \multicolumn{9}{|l|}{ Toma de decisiones. Responsabilidades } \\
\hline \multicolumn{9}{|l|}{ Afrontamiento del cambio } \\
\hline \multirow{2}{*}{\multicolumn{9}{|c|}{ Investigación, diagnóstico y evaluación }} \\
\hline & & & & & & & & Realización y ejecución de propuestas \\
\hline \multirow{2}{*}{\multicolumn{9}{|c|}{$\begin{array}{l}\text { Destrezas comunicativas en lengua propia } \\
\text { Destrezas comunicativas en lenquas extranjeras }\end{array}$}} \\
\hline \multirow{2}{*}{\multicolumn{9}{|c|}{ Destrezas comunicativas en lenguas extranjeras }} \\
\hline & & & & & & & & \\
\hline \multicolumn{9}{|l|}{ Aspectos actitudinales y éticos } \\
\hline \multicolumn{9}{|l|}{ Orientación y Acción tutorial } \\
\hline Gestión y promoción de valores & & & & & & & & \\
\hline
\end{tabular}

b) De las competencias a las atribuye mayor importancia, explique por qué:

c) Añada otras que crea que tiene

d) Sugiera otras

10- De la formación continua recibida, ¿en qué competencia(s) cree que mejora?

A. Competencia científica $\square$ F. Competencia en trabajo en equipo

B. Competencia intra e interpersonal

C. Competencia didáctica

D. Competencia organizativa y de gestión del centro

E. Competencia en gestión de la convivencia

G. Competencia en innovación y mejora

$\mathrm{H}$. Competencia comunicativa y lingüística

I. Competencia digital (TIC)

Otras $\square$ ¿Cuáles?:

J. Competencia social-relacional 
11- Además de la formación continua recibida, ¿qué hace para mejorar sus competencias?

12- En adelante, ¿qué piensa hacer para mejorar esas competencias?

13- ¿Participa en programas de evaluación docente? Sí

No

En caso de Sí, ¿cuáles y con qué frecuencia los utiliza?

14- ¿Pasa pruebas oficiales para actualizar sus certificados de acreditación en Lenguas Extranjeras?

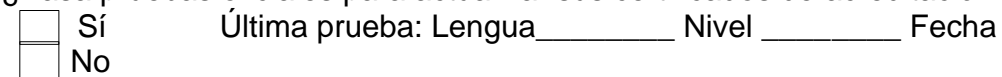

15- Sobre su perfil profesional:
a) ¿Considera que tiene un reconocimiento y una certificación como corresponde? Si
No
b) En caso de Sí, ¿de qué manera se ha resuelto "oficialmente"?
c) ¿Qué utilidad o beneficios ha encontrado?
d) En caso de No, ¿lo considera necesario? Sí $\square$ No
e) ¿Qué expectativas tiene al respecto

16- Conforme a esta, indique el grado de realización de las siguientes acciones:

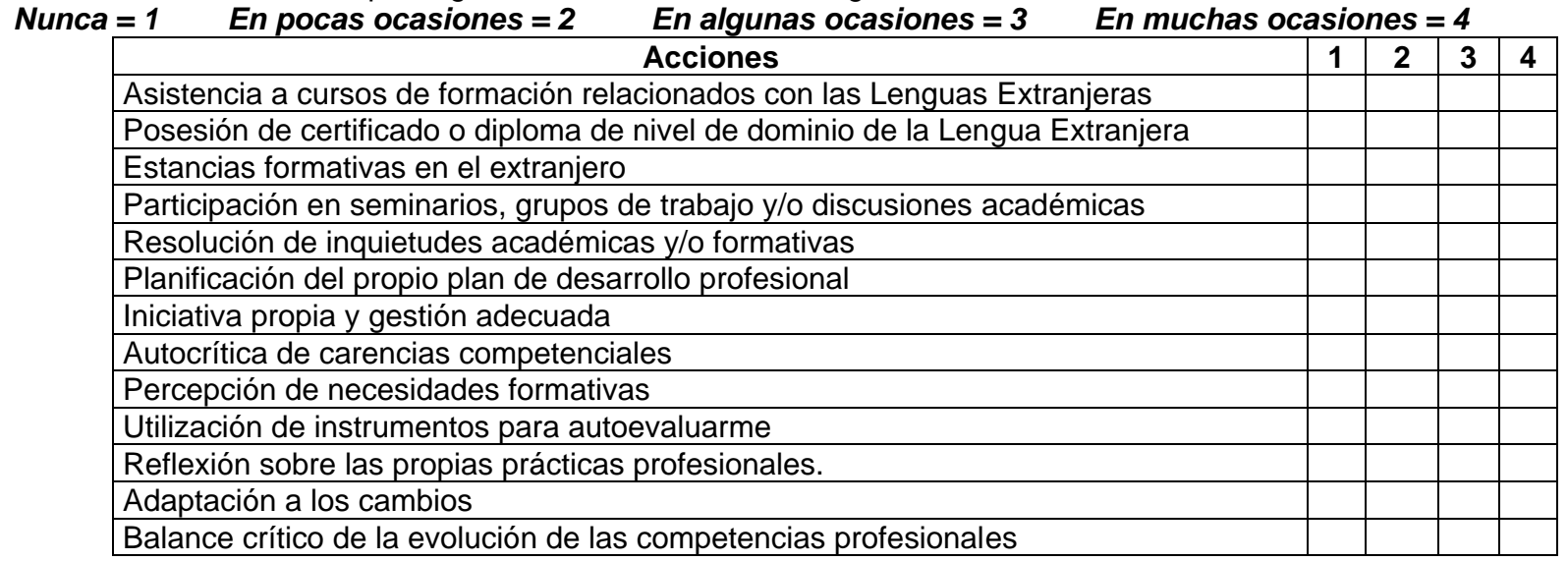

17- Añada otras acciones con disposición para realizarlas:

18-A modo de balance final:

a) Hasta su actual ocupación laboral ha pasado por

b) ¿Por las redes institucionales, encuentra fácilmente actividades y cursos de formación?

Sí $\square$ No

c) Rodee los términos calificarían su experiencia profesional docente:
Motivación
Perspicacia
Compromiso
Condiciones formativas
Deseo de progreso Determinación Toma de conciencia Control
Curiosidad Seguridad
Apertura al desarrollo profesional

Añada otros que se aplicaría:

d) ¿Con este cuestionario toma conciencia de los elementos que le "sitúan" en su carrera profesional y de la importancia de los mismos en su desarrollo profesional? Sí

$\checkmark$ Otros comentarios: 